\section{P123 OPIATE SMOKERS HAVE A HIGH PREVALENCE OF RESPIRATORY SYMPTOMS IRRESPECTIVE OF AIRFLOW OBSTRUCTION}

${ }^{1} \mathrm{~N}$ Lewis-Burke, ${ }^{1} \mathrm{O}$ Wooding, ${ }^{1} \mathrm{~K}$ McKinnell, ${ }^{2} \mathrm{~L}$ Davies, ${ }^{2} \mathrm{PP}$ Walker; ${ }^{1}$ Sefton Primary Care Trust, Liverpool, UK; ${ }^{2}$ University Hospital Aintree, Liverpool, UK

\subsection{6/thoraxjin-2013-204457.273}

In the 1990s inhalation ('chasing the dragon') became the predominant method of recreational opiate (heroin and crack cocaine) consumption as it was perceived to have fewer detrimental health effects than injection. Although clinicians encounter individuals with COPD associated with opiate smoking ('heroin lung') the airway effects and symptoms resulting from opiate smoking are not established. We recruited 145 current and past opiate users from a local community drug service and recorded demographics. They completed spirometry pre and post salbutamol and questionnaires addressing drug use, symptoms and health status. Lower limit of normal was used to define airflow obstruction. Ten subjects failed to produce adequate spirometry, 26 had only injected and never smoked opiates while 6 subjects had marked bronchodilator (BD) reversibility consistent with asthma. Thirty six subjects appeared to have COPD and these were compared with 67 opiate smokers with normal postBD spirometry and the 26 subjects who only injected opiates. The results from the 3 groups are shown in the table. The COPD group was a little older and necessarily had a lower FEV1 and post-BD airflow obstruction. There was little difference in length of drug use when the opiate smokers with COPD were compared to those without and the frequency of cough and phlegm differed little. Opiate smokers with COPD had modestly higher rates of wheeze and breathlessness; hence, higher CAT and MRC dyspnoea scores, but respiratory symptoms and use of respiratory medication were common in the non-COPD groups. Heroin and crack cocaine smoking is a risk factor for the development of irreversible airflow obstruction at a very young age. Respiratory symptoms are common in opiate

\begin{tabular}{lllll} 
Abstract P123 Table 1. & & & & \\
\hline & $\begin{array}{l}\text { COPD } \\
\text { (n=36) }\end{array}$ & $\begin{array}{l}\text { No COPD } \\
(\mathbf{n}=67)\end{array}$ & $\begin{array}{l}\text { No COPD, } \\
\text { IVDU only } \\
(\mathbf{n}=26)\end{array}$ & $\begin{array}{l}\text { p Value } \\
\text { COPD VS. } \\
\text { No COPD }\end{array}$ \\
\hline Age (yrs) & $44.7(6.3)$ & $40.3(7)$ & $34.6(12)$ & 0.01 \\
Gender (Male/\%) & $25(69 \%)$ & $48(72 \%)$ & $16(59 \%)$ & NS \\
Post-BD FEV1 (L) & $2.62(0.91)$ & $3.65(0.82)$ & $3.85(1.18)$ & $<0.001$ \\
Post-BD FEV1 (\%) & $74.6(23.8)$ & $99.2(14.4)$ & $102.7(11.4)$ & $<0.001$ \\
Post-BD FEV1/FVC & $0.6(0.13)$ & $0.77(0.05)$ & $0.84(0.05)$ & $<0.001$ \\
CAT & $19.8(9.8)$ & $16.6(9.5)$ & $10.2(7.4)$ & NS \\
MRC & $3(1.6)$ & $2.3(1.2)$ & $1.4(0.6)$ & 0.06 \\
Using inhalers & $19(56 \%)$ & $24(36 \%)$ & $2(8 \%)$ & NS \\
Using long-acting BD & $9(25 \%)$ & $5(8 \%)$ & 0 & 0.02 \\
Cough (\%) & 67 & 69 & 31 & NS \\
Phlegm (\%) & 69 & 63 & 35 & NS \\
Wheeze (\%) & 86 & 63 & 31 & 0.01 \\
Breathlessness (\%) & 83 & 69 & 23 & NS \\
Cigarette pack years & $24(21)$ & $23(17)$ & $19(19)$ & NS \\
Years heroin smoked & $24(9)$ & $15(10)$ & NA & $<0.001$ \\
Years crack smoked & $13(8)$ & $11(7)$ & NA & NS \\
Years cannabis smoked & $22(11)$ & $18(11)$ & $9(6)$ & 0.08 \\
Diagnosed with asthma (n/\%) & $21(58 \%)$ & $18(27 \%)$ & $4(15 \%)$ & $<0.01$ \\
Diagnosed with COPD (n/\%) & $5(14 \%)$ & $4(6 \%)$ & 0 & NS \\
\hline & & & & \\
\hline
\end{tabular}

smokers irrespective of the presence of COPD and not uncommon in those who have only injected opiates. In many, this is associated with a reduced health status despite normal spirometry; hence, symptoms are not a useful way to 'diagnose' COPD and spirometry is essential.

\section{P124 CHRONIC BRONCHITIS AMONG FISHERMEN IN EXPOSED TO FIREWOOD SMOKE}

VA Umoh; University of Uyo Teaching Hospital, Uyo, Nigeria

\subsection{6/thoraxjnl-2013-204457.274}

Background Biomass is heavily depended on for domestic energy use by people in developing countries. These materials are typically burnt in simple stoves and produce a lot of smoke. Exposure to this indoor air pollution has been linked to a number of respiratory disorders. This study aimed to assess some long term effects exposure to indoor air pollution among fishermen.

Methods A survey was conducted in a fishing community in Nigeria among 337 fishermen exposed to indoor air pollution from burning firewood and 345 matched controls. Exposure was determined by the product of the average daily duration of time spent close to the fire and the number of years (Hour-years). A modified BMRC questionnaire was used to obtain information on respiratory symptoms and spirometry was performed on the participants.

Results The frequency of chronic respiratory symptoms was significantly higher among the exposed fishermen compared with the control subjects. Chronic bronchitis was significantly associated with an obstructive ventilatory pattern. Logistic regression analysis showed an increased risk for chronic bronchitis among exposed fishermen (OR 8.7; 95\% CI 4.7-16.3, p <0.001), women were 6 times more likely than men to develop chronic bronchitis (OR 6.6; 95\% CI 2.5-17.8, p < 0.001) and cigarette smokers were 5 times more likely than non-smokers to develop chronic bronchitis (OR 5.0; 95\% CI 1.8-13.8, p < 0.05).

Conclusion The results of this survey have shown an association between exposure to indoor air pollution and chronic respiratory disorders. Cigarette smoking aggravated this association.

\section{P125 HEROIN SMOKING IS ASSOCIATED WITH EARLY ONSET COPD WITH PREDOMINANT EMPHYSEMA}

PP Walker, E Thwaite, S Amin, J Curtis, PM Calverley; University Hospital Aintree, Liverpool, UK

\subsection{6/thoraxjnl-2013-204457.275}

An association between opiate smoking and acute severe asthma is recognised but a relationship with COPD is not clearly established and limited to case reports. Anecdotally there is limited knowledge about the pulmonary effects of heroin smoking amongst drug users, drug service workers and healthcare professionals. To examine this relationship we studied a convenience sample of 41 heroin smokers who presented to our service with respiratory symptoms and were diagnosed with COPD. All completed spirometry and underwent a CT scan while 20 had alpha1-antitrypsin level measured and 9 had DLco measured. Visual emphysema extent was recorded by two thoracic radiologists with a third adjudicating where there was a more than 1 point difference in grading. Emphysema was assessed in both lungs at 